\title{
Secondary Hemophagocytic Lymphohistocytosis in COVID-19
}

\author{
Stephanie Lilia Pushpam Jetty ${ }^{1}$, Dotton Denis Noronha ${ }^{2 *}$, Akshatha Nayak ${ }^{3}$ and Altin Dsouza ${ }^{4}$ \\ 'Department of Pathology, Father Muller Medical College, Kankanady, Mangalore, Karnataka, India, 575002 \\ ${ }^{2}$ Department of Critical care \& Internal medicine, A.J. Hospital \& Research Centre, Kuntikana, Mangalore, Karnataka, India, 575004 \\ ${ }^{3}$ Department of Hematology, A.J. Hospital \& Research Centre, Kuntikana, Mangalore, Karnataka, India, 575004 \\ ${ }^{4}$ Department of Respiratory Therapy, A.J. Hospital \& Research Centre, Kuntikana, Mangalore, Karnataka, India, 575004
}

\begin{abstract}
We present case of a 53-year-old lady who is a known case of rheumatoid arthritis and hypertension in Karnataka, South India, who presented with Severe Acute Respiratory Infection (SARI) like symptoms (fever, cough and breathlessness) due to COVID-19. During the course of treatment further investigations revealed evidence of Hemophagocytic Lymphohistocytosis (HLH). This case emphasizes the need for a thorough workup to identify the etiology of HLH as it may unmask a treatable entity in a COVID-19 patient.
\end{abstract}

Keywords: Hemophagocytic lymphohistiocytosis, COVID-19, Severe Acute Respiratory Infection

\section{Introduction}

It is known that COVID-19 can cause an intense cytokine mediated immune response causing various complications acute respiratory distress syndrome. However, one extreme of such a strong immune response is Hemophagocytic Lymphohistocytosis (HLH). HLH being a rare condition in itself, is lately diagnosed and it mimics the cytokine storm in COVID-19.

\section{Case Report}

We present the case of a 53-year-old female, with history of hypertension \& rheumatoid arthritis, taking cilnidipine daily, leflunomide daily \& methotrexate once in 7 days with no history of cigarette or alcohol consumption and no known drug allergies.

The patient came to seek medical care at A.J. Hospital \& Research Centre on 7/10/2020 following 10 days treatment (antibiotics and Remdesivir ) at another hospital for RTPCR proven nasopharyngeal swab for SARS-Cov2 RNA virus, which was diagnosed on 1/10/2020. She reported that the reason for seeking medical care was the worsening of fever \& dyspnoea on $7 / 10 / 2020$. On general physical examination, patient was extremely tachypnoeic, spiking temperature of $100{ }^{\circ} \mathrm{F}$ with a respiratory rate of $38 \mathrm{rpm}$, when breathing on room air. She was maintaining an oxygen saturation of $77 \%$, despite on 15 litres of High Concentration mask. Respiratory examination revealed bilateral crackles in the lower and middle segments of lungs. In the background of swab positive RT-PCR 1 week back, the possible differential diagnosis was COVID -19 associated Acute respiratory distress syndrome, possibly in a cytokine storm phase, so a repeat nasopharyngeal swab specimen was collected along with other routine investigations - complete blood cell count, blood chemistry, inflammatory markers (IL-6, D-Dimer, ferritin, LDH, CRP). (see table 1)

The patient was shifted to COVID intensive care unitand high flow nasal oxygen (HFNO) was offered, thereby improving her oxygen saturation to $94 \%$ and was encouraged to do awake proning as well as Incentive Spirometry. Apart from her antihypertensives and disease modifying anti rheumatic drugs (DMARDs), she was started on - Meropenem, Voriconazole, Oseltamivir, Ivermectin, Low Molecular Weight Heparin- Enoxaparin sodium (60mg subcutaneous bid), Methylprednisolone (120mg/ day), Mucolytics, vitamin $\mathrm{C} \&$ zinc and 2 doses of tocilizumab(400mg) were initiated on $7 / 10 / 2020$ in view of elevated inflammatory markers. A baseline 2d echocardiography was done and it revealed normal ventricular functions with no pulmonary artery hypertension and chest computed tomography (CT) reported as multiple patchy ground glass opacities with consolidation and fibrotic bands involving bilateral lungs, predominantly the mid and lower lobes- CORAD 6 with CT severity index 23/25.

The initial 10 days of treatment, she was comfortable on high flow nasal oxygen (HFNO) and her oxygen requirement was being tapered, with routine awake pruning and incentive spirometry, though her inflammatory markers were worsening with no significant improvement on serial chest X-rays. On 21/10/2020, she started deteriorating on HFNO, requiring a higher concentration of oxygen day- 
Table 1: Main lab results of the patient.

\begin{tabular}{|c|c|c|c|c|c|c|c|}
\hline & $9 / 10 / 2020$ & $26 / 10 / 2020$ & $29 / 10 / 2020$ & $30 / 10 / 2020$ & $31 / 10 / 2020$ & $1 / 11 / 2020$ & $2 / 11 / 2020$ \\
\hline $\mathrm{HB}(\mathrm{g} / \mathrm{dl})$ & 9 & 11.3 & 10.4 & 11.7 & 7.7 & 10.6 & 12.7 \\
\hline $\mathrm{TC}(/ \mu \mathrm{l})$ & 6,300 & 8640 & 5530 & 2410 & 320 & 480 & 50 \\
\hline Neutrophils \% & 95.7 & 95.4 & & & 88 & 85 & \\
\hline Lymphocytes \% & 3 & 1.5 & & & 10 & 10 & \\
\hline Monocytes \% & 1.3 & 3 & & & 2 & 5 & \\
\hline Eosinophils \% & 0 & 0 & & & 0 & 0 & \\
\hline Basophils \% & 0 & 0.1 & & & 0 & 0 & \\
\hline ANC $(/ \mu \mathrm{l})$ & 6103 & & & & & 410 & 0 \\
\hline $\operatorname{ALC}(/ \mu \mathrm{l})$ & 190 & & & & & 50 & 0 \\
\hline AMC $(/ \mu \mathrm{l})$ & 80 & & & & & 20 & 0 \\
\hline $\mathrm{AEC}(/ \mu \mathrm{l})$ & 0 & & & & & 0 & 0 \\
\hline $\mathrm{ABC}(/ \mu \mathrm{l})$ & 0 & & & & & 0 & 0 \\
\hline $\mathrm{nRBC}$ & 0 & & & & & 90 & 440 \\
\hline Platelets $(/ \mu \mathrm{l})$ & $2,41,000$ & $1,10,000$ & 75,000 & & 65,000 & 40,000 & 50,000 \\
\hline Procalcitonin & & & & & 0.32 & & \\
\hline s. Albumin & & & & & 2.3 & & 3.1 \\
\hline Total bilirubin $(\mathrm{mg} / \mathrm{dl})$ & 0.5 & 0.8 & & & 2.2 & 2.9 & 3.2 \\
\hline Direct bilirubin (mg/dl) & 0.3 & 0.4 & & & 1.5 & 2.1 & 1.5 \\
\hline SGOT (IU/L) & 40 & 61 & & & 112 & 3.3 & 65 \\
\hline SGPT (IU/L) & 72 & 149 & & & 307 & 109 & 121 \\
\hline s. LDH (U/I) & 510 & 1095 & & & 840 & 266 & 724 \\
\hline $\mathrm{CRP}(\mathrm{mg} / \mathrm{L})$ & 78.10 & 0.66 & & & & & \\
\hline IL-6 (pg/ml) & 218.70 & & & & & & \\
\hline s. Ferritin & $\begin{array}{l}>2000 \\
(4659.88)\end{array}$ & $\begin{array}{l}>2000 \\
(4970.46)\end{array}$ & & & $\begin{array}{l}>2000 \\
(5969.99)\end{array}$ & & \\
\hline D-Dimer (ng/ml) & 243 & 1116 & & & 498 & & \\
\hline $\begin{array}{l}\text { Fibrinogen } \\
\text { (mg/dl) }\end{array}$ & & & & & 140 & & \\
\hline $\begin{array}{l}\text { s. Triglyceride } \\
(\mathrm{mmol} / \mathrm{l})\end{array}$ & & & & & 226 & & \\
\hline RBC folate level (ng/ml) & & & & & 386 & & \\
\hline s. folic acid (ng/ml) & & & & & 10 & & \\
\hline Vitamin B12 (pg/ml) & & & & & $\begin{array}{l}>2000 \\
(4781)\end{array}$ & & \\
\hline ANA & & & & & $\begin{array}{l}<0.5 \text { index } \\
\text { Negative }\end{array}$ & & \\
\hline DCT & & & & & Negative & & \\
\hline
\end{tabular}




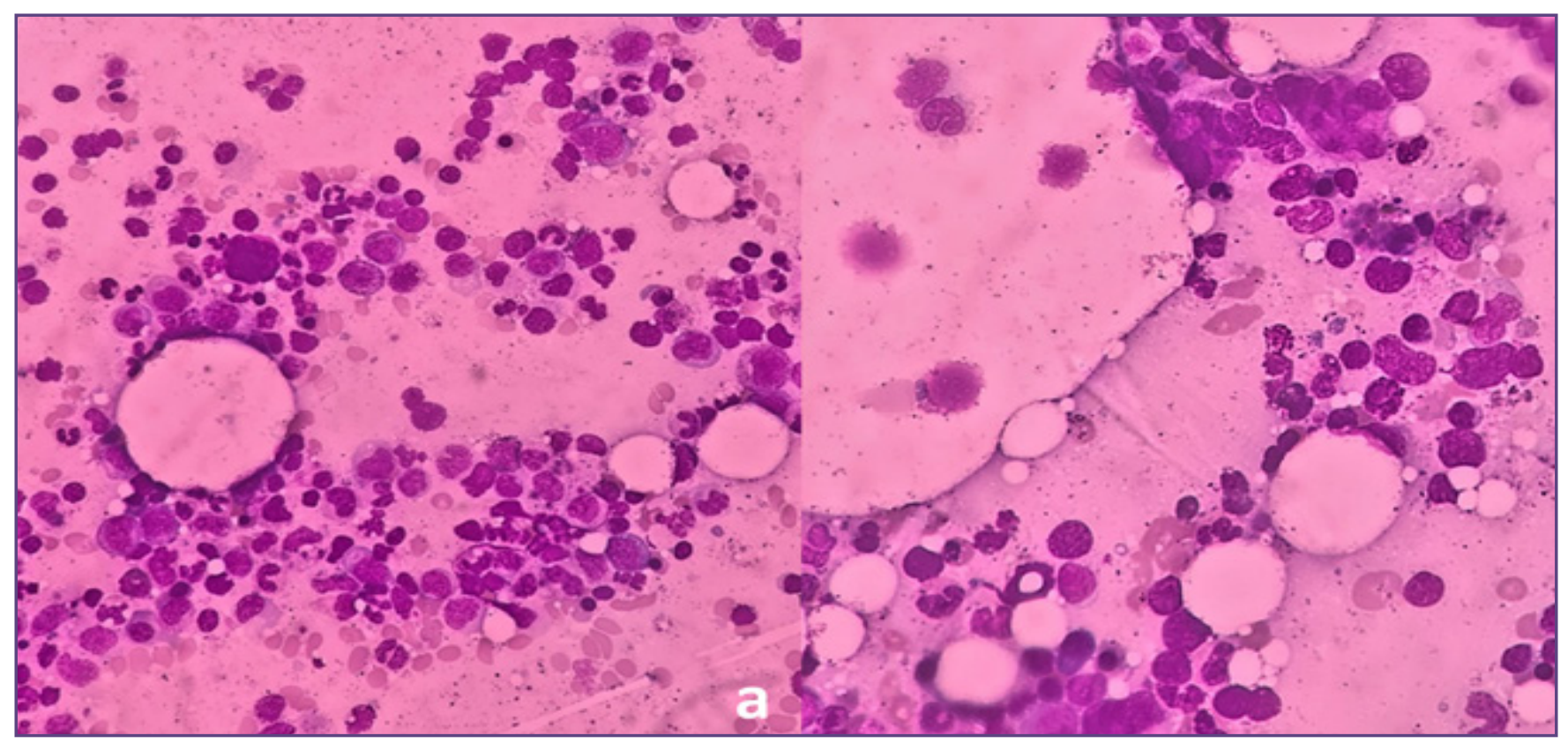

Fig. 1: a and b, Bone Marrow Aspirate smear demonstrating macrophage with nuclear remnants of ingested erythrocytes and leukocytes. MGG x 40.

by-day. Non- Invasive ventilation (NIV) was started on 25/10/2020 due to sudden oxygen desaturation despite on maximum settings on HFNO. However, due to persistent desaturation despite NIV, patient was electively intubated using an Hi-Lo Evac endotracheal tube with a Macintosh laryngoscope blade, sedated with fentanyl and vecuronium; and connected to invasive mechanical ventilation; central venous catheterisation was done using right jugular vein for venous access, an arterial line was placed using right ulnar access for mean arterial pressure (MAP) monitoring as patient was on inotropic supports ( dopamine and norepinephrine); a urinary catheter for output monitoring and a naso-gastric tube was inserted for enteral feeding. Patient was receiving stepwise lung recruitment manoeuvres every 2 nd hourly in view of poor lung compliance and severe arterial hypoxia. Patient was put on prone ventilation every 16 hours with sedation vacation trials daily when in supine position. The following tests were ordered serially on day-to-day basis: chest $\mathrm{x}$-ray, complete blood counts, arterial blood gases, procalcitonin levels and inflammatory markers. Blood and endotracheal cultures were negative.

On 28/10/2020, patients right lower limb was noticed to be swollen, and venous doppler study revealed deep vein thrombosis in right lower limb venous system involving common femoral, superficial femoral, popliteal, anterior tibial and posterior tibial veins. As inflammatory markers were rising and $2 \mathrm{~d}$ echocardiography started showing increase in pulmonary artery hypertension (PASP by TR jet $40 \mathrm{mmHg}$ ), a CT-Pulmonary angiogram was done to look for any peripheral thrombus, and it was reported as severe pulmonary artery hypertension. Considering this, the dose of Enoxaparin sodium was increased from $60 \mathrm{mg}$ to $90 \mathrm{mg}$ subcutaneous and then eventually was switched over to newer oral anticoagulant -Dabigatran (150mg bid) and Inhaled nitric oxide (INO) therapy was initiated. Empirically Teicoplanin was added to meropenem for both gram negative and positive coverage in view of worsening hemodynamics, and Methylprednisolone(120mg/day) with fentanyl-midazolam/fentanyl-vecuronium infusion were continued.

0n 29/10/2020, patient developed rectal bleeding. Per rectal examination revealed fresh blood not mixed with stools. On sigmoidoscopy, it was found that distal rectum showed multiple ulcers - Stercoral ulcers. Patient was treated with proctolysis enema and was on liquid paraffin for the same following which there was no rectal bleed noticed.

On 31/10/2020, patients laboratory values showed pancytopenia with very severe neutropenia with haemoglobin $7.7 \mathrm{~g} / \mathrm{dl}$, platelets $65,000 / \mu 1$, total counts 320/ $\mu$, absolute neutrophil count (ANC) 280 (corrected for nRBCs),serum ferritin 5969, D-dimer $400 \mathrm{ng} / \mathrm{ml}$, procalcitonin 0.3, LDH $840 \mathrm{U} / 1$, total bilirubin $2.2 \mathrm{mg} /$ $\mathrm{dl}$, direct bilirubin $1.5 \mathrm{mg} / \mathrm{dl}$, with a normal creatinine level $0.8 \mathrm{mg} / \mathrm{dl}$, serum calcium $7 \mathrm{mg} / \mathrm{dl}$. Peripheral smear showed pancytopenia with no abnormal or atypical cells. Since patient was on DMARDs, vitamin B12 and folate 
levels were sought, to rule out drug induced causes of pancytopenia. Vitamin B12 levels were $>2000 \mathrm{pg} / \mathrm{ml}$ (1:3 Dilution:4781) and $\mathrm{RBC}$ folate level were $386 \mathrm{ng} /$ $\mathrm{ml}$. Direct Coombs test (DCT) was asked in view of elevated LDH and mild increase in bilirubin levels, which was found to be negative. Anti-nuclear antibody (ANA) immunofluorescence test was done to rule out autoimmune cytopenia and was found to be negative. Viral PCR (CMV, EBV, Adenovirus, Parvovirus) were sent to rule out infectious causes of pancytopenia, which were found to be negative. Repeat Blood cultures (aerobic \& anaerobic) from both central and peripheral vessels were sent for culture and sensitivity.

HLH work up was done where serum ferritin was $>2000$, serum fibrinogen levels were $140 \mathrm{mg} / \mathrm{dl}$, serum triglyceride levels were $226 \mathrm{mmol} / \mathrm{l}$ despite patient was on methylprednisolone $(120 \mathrm{mg} /$ day $)$ from first day of admission. Therapy wise 1 packed cells RBCs was transfused, methylprednisolone $(120 \mathrm{mg} /$ day $)$ was continued, antibiotics stepped up from meropenem to colistin and Fosfomycin therapy in view of severe febrile neutropenia, and antifungals (anidulafungin) were prophylactically given. 1 dose of granulocyte colony stimulating factor (G-CSF) was administered and methotrexate (last dose 25/10/2020) and Leflunomide were withheld.

On 1/11/2020, 12 hours post G-CSF, complete blood count showed an increase in ANC and she was put on G-CSF twice daily regimen. Tissue diagnosis of HLH in the form of bone marrow biopsy was done and it demonstrated macrophage with nuclear remnants of ingested erythrocytes and leukocytes (Fig.1, Fig.2) and the diagnosis of HLH was made. In view of prolonged ventilation, patient was decided to put on veno-venous ECMO and during the procedure 4 packed cell RBCs and 10 units random donor platelets were transfused and heparin infusion was kept to maintain activated clotting time $150-170$ and all other anticoagulation was withheld.

On 2/11/2020, patient's haemoglobin was $12.4 \mathrm{~g} / \mathrm{dl}$, total counts $50 / \mu 1$, platelets $49,000 / \mu 1$. Patient had a cardiac arrest the same afternoon and was decided to put on veno-arterial ECMO as well. In the presence of repeated swab (RT-PCR, CB-NAAT, RAT) positive test, a negative procalcitonin level and positive laboratory and tissue biopsy diagnostic of HLH in the presence of fever, patient was declared dead on 3/11/2020 at 2.05am secondary to HLH in COVID-19 despite on methylprednisolone ( $120 \mathrm{mg} /$ day $)$.

\section{Discussion}

Secondary HLH is similar to primary HLH, but is characterized by lack of genetic defects, and is triggered by events that disrupt immune balance. HLH is further subclassified into infection-related, malignancy related and associated forms of inflammation / autoimmunity. Secondary HLH is also seen in immunodeficiency following chemotherapy, immunosuppression, biology therapy and organ or stem cell transplantation, insults causing imbalance to the immune system. ${ }^{[1-3]}$ Abnormal cases are also described in birth disorders such as lysinuric protein incompatibility or biotinidase deficiency. Secondary HLH in metabolic patients is usually atypical, deficient in fever or incorporates non-HLH factors such as vomiting or acidosis. ${ }^{[2]}$

Around $60 \%$ of infectious cases are accompanied with primary infection or reactivation of latently present herpesviruses. ${ }^{[3]} \mathrm{EBV}$ infects B cells and nasopharyngeal epithelial cells, but in HLH it often infects CTLs and NK cells. ${ }^{[4]}$ Other viruses that are known to trigger HLH are -Cytomegalovirus, herpes simplex virus (HSV), human herpesvirus- 6 and -8 , varicella zoster, parvovirus B19 and adenoviruses. HLH is less frequently seen in RNA viruses (influenza and enteroviruses). Patients suffering human immunodeficiency virus (HIV) infection are prone to develop HLH upon opportunistic infections. ${ }^{[3,5,6]}$

Secondary HLH also occurs in autoimmune and autoinflammatory disorders like systemic lupus erythematosus, adult-onset Still's disease, Kawasaki disease, rheumatoid arthritis, dermatomyositis, sarcoidosis, systemic sclerosis, polyarteritis nodosa, inflammatory bowel disease and systemic juvenile idiopathic arthritis. Complicated rheumatological conditions with HLH is termed as 'macrophage activation syndrome' (MAS). ${ }^{[3,7,8]}$

In secondary HLH, the NK cell dysfunction is acquired. It is a temporary phenomenon that often normalizes with disease remission and reverses upon IL2 stimulation in vitro. [9,10,11,12] The cytokine storm is considered to impair NK cell cytotoxicity. Persistent high levels of NK cell-stimulating cytokines (IL12 or IL18), might over activate NK cells, leading to apoptosis. ${ }^{[13,14]}$

\section{Conclusion}

Secondary HLH has also been found in patients with severe acute respiratory syndrome and middle east respiratory syndrome (MERS). It is likely that it develops in patients suffering from COVID-19 too, as all three viruses trigger a similar cytokine mediated storm. It is probably existing in more patients than we know with COVID-19 and likely contributes to prognosis and its mortality and needs to be thoroughly evaluated for the same as its a treatable entity.

\section{Funding}

None 


\section{Competing Interests}

None declared

\section{References}

1. Atteritano, M., David, A., Bagnato, G., Beninati, C., Frisina, A., Iaria, C. \& Cascio, A. (2012) Haemophagocytic syndrome in rheumatic patients. A systematic review. European Review for Medical and Pharmacological Sciences, 16, 1414- 24.

2. Janka, G.E. \& Lehmberg, K. (2014) Hemophagocytic syndromes - An update. Blood Reviews, 28, 135- 142.

3. Ramos-Casals, M., Brito-Zerón, P., López-Guillermo, A., Khamashta, M.A. \& Bosch, X. (2014) Adult haemophagocytic syndrome. Lancet, 383, 1503-16.

4. Kasahara, Y., Yachie, A., Takei, K., Kanegane, C., Okada, K., Ohta, K., Seki, H., Igarashi, N., Maruhashi, K., Katayama, K., Katoh, E., Terao, G., Sakiyama, Y. \& Koizumi, S. (2001) Differential cellular targets of Epstein-Barr virus (EBV) infection between acute EBV-associated hemophagocytic lymphohistiocytosis and chronic active EBV infection. Blood, 98, 1882- 1888.

5. Rouphael, N.G., Talati, N.J., Vaughan, C., Cunningham, K., Moreira, R. \& Gould, C. (2007) Infections associated with haemophagocytic syndrome. The Lancet. Infectious diseases, 7, 814-822.

6. George, M.R. (2014) Hemophagocytic lymphohistiocytosis: review of etiologies and management. Journal of Blood Medicine, 5, 69-86.

7. Atteritano, M., David, A., Bagnato, G., Beninati, C., Frisina, A., Iaria, C. \& Cascio, A. (2012) Haemophagocytic syndrome in rheumatic patients. A systematic review. European Review for Medical and Pharmacological Sciences, 16, 1414- 24.
8. Zhang, M., Behrens, E.M., Atkinson, T.P., Shakoory, B., Grom, A.A. \& Cron, R.Q. (2014b) Genetic defects in cytolysis in macrophage activation syndrome. Current Rheumatology Reports, 16, 439-446.

9. Grom, A., Villanueva, J., Lee, S., Goldmuntz, E., Passo, M. \& Filipovich, A.H. (2003) Natural killer cell dysfunction in patients with systemic-onset rheumatoid arthritis and macrophage activation syndrome. Journal of Pediatrics, 29, 2- 296.

10. Ramanan, A.V. \& Schneider, R. (2003) Macrophage activation syndrome-what's in a name!. The Journal of Rheumatology, 30, 2513-6.

11. Grom, A.A. (2004) Natural killer cell dysfunction: a common pathway in systemic-onset juvenile rheumatoid arthritis, macrophage activation syndrome, and hemophagocytic lymphohistiocytosis? Arthritis and Rheumatism, 50, 689-98.

12. Bryceson, Y.T., Pende, D., Maul-Pavicic, A., Gilmour, K.C., Ufheil, H., Vraetz, T., Chiang, S.C., Marcenaro, S., Meazza, R., Bondzio, I., Walshe, D., Janka, G., Lehmberg, K., Beutel, K., zur Stadt, U., Binder, N., Arico, M., Moretta, L., Henter, J.I. \& Ehl, S. (2012) A prospective evaluation of degranulation assays in the rapid diagnosis of familial hemophagocytic syndromes. Blood, 119, 2754- 2763.

13. Avau, A., Put, K., Wouters, C.H. \& Matthys, P. (2015) Cytokine balance and cytokine-driven natural killer cell dysfunction in systemic juvenile idiopathic arthritis. Cytokine \& Growth Factor Reviews, 26, 35- 45.

14. Canna, S.W. \& Goldbach-Mansky, R. (2015) New monogenetic autoinflammatory diseases-a clinical overview. Seminars in Immunopathology, 37, 387- 394.

*Corresponding author:

Dr. Dotton Denis Noronha, Consultant Intensivist \& Assistant Professor, Department of Critical care \& Internal medicine, A.J. Hospital \& Research Centre, Kuntikana, Mangalore, Karnataka, India, 575004

Phone: +91 8095241384

Email: dottonnoronha@gmail.com

Date of Submission $\quad: 27 / 01 / 2021$

Date of Final Revision : 13/02/2021

Financial or other Competing Interests: None. 